\title{
Der Sachsenspiegel und das Magdeburger Recht in Weißrussland. Konkrete Anwendung in der Rechtspraxis
}

\author{
Olga Keller
}

Karl Eberhard University of Tübingen, Philosophical Faculty

Belarusian State University of Minsk, Historical Faculty

E-mail:olga.keller@histsem.uni-tuebingen.de,olga.keller@mail.ru

\section{The Saxon Mirror and Magdeburg Law in Belarus. Concrete Application in Legal Practice}

\begin{abstract}
:
The article focuses on further research into the source situation. It tries to find out whether German law (Saxon Mirror and Magdeburg city law) was merely transferred to Belarus, or whether it was also received by Belarusian towns and villages into their practice. If this is the case, the question is where it can be seen, and by which sources it can be proven. An attempt to investigate the concrete application of Saxon Mirror and Magdeburg city law follows, with focus on different forms in legal practice, presented on specific cases. It must be stressed that the very extensive research into legal practice has been carried out only by me so far and will be the aim of my future research. In line with the findings of my research stay, my research report is divided into three parts: I. The National Archives of the Republic of Belarus in Minsk; II. The National Library of Belarus in Minsk; III. The National Museum in the City of Nesvish.
\end{abstract}

Key words: Saxon Mirror; Magdeburg city law; application of German law; reception; transfer; documents of the National Historical archive of Belarus; sources from the National Library of Belarus; museum in the town hall in Nesvish; historical and legal materials; acts of legal nature; specific cases of legal practice; magdeburgii

DOI: $10.14712 / 2464689 X .2021 .2$ 
Das Thema meines Aufsatzes heißt »Sachsenspiegel und Magdeburger Recht in Weißrussland. Konkrete Anwendung in der Rechtspraxis« und basiert auf den Ergebnissen meines letzten Forschungsaufenthalts in Weißrussland. In meinen Vorarbeiten habe ich aufgezeigt, dass das deutsche Recht in Form des Sachsenspiegels und des Magdeburger Stadtrechts mit seinen Varianten in den weißrussischen Ortschaften des Großfürstentums Litauen und des Königreichs Polen (z.B. in Brest im Jahre 1390, in Grodno im Jahre 1391 usw.) verbreitet war. ${ }^{1}$

Die Schwerpunkte dieses Artikels liegen auf der weiteren Erforschung der Quellenlage. Ich habe versucht herauszufinden, ob das deutsche Recht (Sachsenspiegel und Magdeburger Stadtrecht) nicht lediglich nach Weißrussland transferiert wurde, sondern ob es in weißrussischen Städten und Dörfern rezipiert wurde. Wenn dies zutreffen sollte, stellt sich die Frage, wo und an welchen Quellen man dies erkennen kann. Daraus folgt der Versuch, die konkrete Anwendung des Sachsenspiegels und des Magdeburger Stadtrechts mit ihren unterschiedlichen Formen in der Rechtspraxis, insbesondere anhand von konkreten Fällen, zu untersuchen. Dabei muss betont werden, dass die sehr umfangreiche Erforschung der Rechtspraxis bisher nur in Ansätzen von mir geleistet werden konnte und Ziel meiner zukünftigen Forschungsarbeit sein wird.

Den Erkenntnissen meines Forschungsaufenthalts entsprechend, gliedert sich mein Forschungsbericht in folgende drei Teile: I. Das Nationalarchiv der Republik Belarus in Minsk; II. Die Nationalbibliothek der Republik Belarus in Minsk; III. Das Nationalmuseum in der Stadt Neswisch.

\section{Die Urkunden des Historischen Nationalarchivs von Weißrussland hinsichtlich der Geschichte des Magdeburger Rechts auf dem Territorium der weißrussischen Länder des Großfürstentums Litauen und des Königreichs Polen}

Das Historische Nationalarchiv Weißrusslands ist eine der größten Schriftgutablagen für die Geschichte Weißrusslands im Zeitraum vom Ende des 14. Jahrhunderts bis Anfang des 20. Jahrhunderts. Das Gründungsdatum ist der 05. Juli 1938. Das Nationalarchiv befindet sich in Minsk. Hier werden die Urkunden für die Geschichte der Periode der Eingliederung Weißrusslands in das Großfürstentum Litauen, in Polen (Rzeczpospolita) und in das

1 KELLER, O. Geschichte, Quellen und Literatur des Magdeburger Rechts in weißrussischen Ortschaften des Großfürstentums Litauen. In: EICHLER, E. - LÜCK, H. Rechts- und Sprachtransfer in Mittel-und Ost-europa: Sachsenspiegel und Magdeburger Recht: intern. u. interdisziplinäre Konf., Leipzig, 31. Okt.-2. Nov. 2003. Berlin: De Gruyter, 2008, S. 103-140. Ivs saxonico-magdebvrgense in Oriente; Bd. 1. Meine anderen deutschsprachigen Aufsätze zum Thema »Das deutsche Recht im Osten« findet man: 1) in der »Zeitschrift der Savigny-Stiftung für Rechtsgeschichte« (2012, Jahrgang 129, S. 364-377). Die Zeitschrift enthält meinen Forschungsbericht unter dem Titel »Aus dem östlichen Europa stammende Forschungsansätze zum Transfer des deutschen Rechts nach Osten in Mittelalter und Neuzeit«; und 2) in der »Zeitschrift für Neuere Rechtsgeschichte« für das Jahr 2012. Diese Zeitschrift publizierte meinen Artikel unter der Bezeichnung »Einführung und Adaption des deutschen Rechts im östlichen Europa während Mittelalter und Früher Neuzeit unter besonderer Berücksichtigung neuerer Forschungen«. Außerdem schreibe ich an einer Habilitation, in der es um den »Transfer des deutschen Rechts nach dem östlichen Europa im Mittelalter und in der Frühen Neuzeit« geht. In meiner Habilitationsschrift werden verschiedene historische Regionen Mittel- und Osteuropas im Mittelalter und in der Frühen Neuzeit auf das Vorhandensein von fünf Elementen des mittelalterlichen deutschen Rechts untersucht, und zwar des Lübecker Stadtrechts, des Magdeburger Stadtrechts, des Nürnberger Stadtrechts, des Sachsenspiegels und des Schwabenspiegels. 
Russische Reich aufbewahrt. Das Archiv umfasst 3.118 Bestände, 29 Mikrofilmsammlungen, zusammen 1.017.136 Einheiten.

Der Dokumentenkomplex der Periode des Großfürstentums Litauen und der Rzeczpospolita beinhaltet die Urkunden des 14.-17. Jahrhunderts, die in altweiß-russischer, altpolnischer und lateinischer Sprache geschrieben sind. Die im Archiv aufbewahrten Urkunden sind Schenkungsurkunden von litauischen Fürsten und polnischen Königen sowie Urkunden von diplomatischer und innerstaatlicher Bedeutung. Darunter befinden sich auch das Privileg nach Magdeburger Recht des Fürsten Nikolaus Christoph Radziwill Sierotka, das am 18. Juli 1586 der Stadt Neswisch verliehen wurde, und ein Erlass (Universal) des Hetman Bogdan Chmelnitzki an die Kaufleute der Stadt Slutzk über Gewährung eines sicheren und freien Handels auf dem Territorium der Ukraine vom 07. Mai 1656.

Zum Stolz des Archivs gehören die Urkundenbücher, in denen die Rechtsakte des Landgerichts Drogitschin von 1416, die Materialien von Magistraten, von Stadt-, Land-, Kaptur-, Oberverwaltungs- (Podkomorski-) Gerichten, von kompromissarischen (Schiedsgerichten), konföderatischen/iven und sonstigen Gerichten, von ordentlichen Zivil- und Militärkommissionen, königlichen Ökonomien, des Krontribunals der Rzeczpospolita und der Haupt- und Staatsgerichtshöfe des Großfürstentums Litauen enthalten sind. Diese Bücher beinhalten die Urkunden über die Wahl der Adelsmarschälle (povetovych marschalkov) und der Beisitzer von Gerichten; die Privilegien polnischer Könige und litauischer Fürsten (in Kopien) über die Gewährung des Magdeburger Rechts an Städte und Ortschaften (1565-1798); die Schenkungsurkunden und Privilegien (in Kopien) von Königen und Fürsten an Magnate, Geistliche, Klöster, Dörfer und katholische Kirchen über Landgüter, Dörfer und Bauern; die Inventurlisten von Städten, Landgütern, Dörfern und Vorwerken mit Aufzählung der Pflichten und Beden (Abgaben) der Bauern; die Erlasse über die Feststellung der Handelsordnung in den Städten, Ortschaften, Dörfern sowie über die Prüfung des Maßes und Waagen; eine Eingabe von Kaufleuten über die Schwierigkeiten der Warenbeförderung auf dem Fluss Düna nach Riga und über die Notwendigkeit eines Dammbaues (1789-1792) u.a.m.

Im Archiv gibt es auch einige Angaben über Baudenkmäler, z.B. die Rathäuser in Minsk und Orscha, oder über die Benennung (Toponymik) der weißrussischen mittelalterlichen Städte.

Das Archiv verfügt über Familienbestände und Urkunden der persönlichen Abstammung, darunter diejenigen der Adelsfamilien Gorwatt, Plater-Sieberg, Radziwill u.a. Im Familienbestand der Fürsten Radziwill sind die auf einem Pergament-blatt gedruckten Schenkungs- und Bestätigungsurkunden für das 16. und 17. Jahrhundert aufbewahrt. Unter den Archivakten existieren auch Materialien über die Geschichte solcher Städte wie Borisov, Witebsk, Minsk, Moghilew, Neswisch, Slutzk u.a.; ebenso über den Stand des Handels, die medizinische Betreuung; über die Kulturgeschichte, die Lebensweise und die Sitten; über die Siegel und Stempel verschiedener Behörden sowie über die Staatsmänner und Persönlichkeiten des öffentlichen Lebens und über sonstige Personen. Neben den dokumentarischen Urkunden befinden sich im Archivbestand auch Dokumente aus der Neswischer Bibliothek der Fürsten Radziwill: Drucksachen und Manuskripte, Noten von Musikwerken, die vollständigen Jahrgänge von Zeitschriften und Zeitungen in englischer, französischer, deutscher, polnischer und russischer Sprache aus dem 18. und 19. Jahrhundert. 
Im Archiv werden verschiedene Sammlungen verwahrt: die Mikrofilmkopien von Urkunden über das sozialökonomische, politisch-gesellschaftliche und kulturelle Leben Weißrusslands vom 16. bis zum Anfang des 20. Jahrhunderts, die aus den staatlichen Archiven von Russland, Litauen und Lettland, aus dem Archiv der alten Hauptakten der Stadt Warschau (Polen), aus den Beständen der Nationalbibliothek der Republik Belarus usw. stammen.

Zur Forschung über das Verbreitungsgebiet des deutschen Rechts auf dem Territorium der weißrussischen Länder, des Großfürstentums Litauen und der Rzeczpospolita können folgende Bestände, Sammlungen und andere Materialien genutzt werden:

\section{Bestände der städtischen Selbstverwaltungsorgane und Gerichtsbehörden}

\section{a) Magistrate}

Der Magistrat ist ein gewähltes Verwaltungs- und Wirtschaftsorgan der Selbstverwaltung in den königlichen und Adelsstädten, die ein Recht auf Selbstverwaltung hatten. Die Magistrate wurden im Großfürstentum Litauen am Ende des 15. Jahrhunderts eingeführt. In den Großfürstenstädten bestanden sie aus zwei Kollegien - dem Stadtrat (Rada) und dem Schöffengericht (Lawa). Der Stadtrat beschäftigte sich mit den Angelegenheiten der Stadtverwaltung und des Gerichts für Vermögens- und Zivilsachen von Bürgern. Das Schöffengericht war zuständig für Strafsachen von Bürgern. An der Spitze des Stadtrats und des Schöffengerichts stand der Vogt. Aufgrund königlicher Privilegien waren der Stadtrat und das Schöffengericht in einigen Städten vereint und handelten in gemeinsamen Sitzungen. Der Magistrat fungierte auch als Notariat. Er setzte sich aus dem Vogt, dem Bürgermeister, den Ratsleuten, den Schöffen und einem Schreiber zusammen. In Weißrussland existierten bis zum Ende des 18. Jahrhunderts 50 Magistrate auf der Grundlage des Magdeburger Rechts; 46 davon in den weißrussischen Städten. Sie wurden nach der Angliederung Weißrusslands an das Russische Reich in Übereinstimmung mit den Erlassen der Zarin Katharina II. abgeschafft. In Ostweißrussland fungierten sie seit 1773, in Zentral- und Westweißrussland seit 1795 als Magistrate. Sie wurden infolge eines Erlasses des Senats im Jahre 1866 aufgelöst.

\section{b) Stadt- und Burggerichte}

Die Stadt- und Burggerichte wurden im 15. Jahrhundert in jedem Kreis gegründet. An der Spitze standen die Ältesten und die Unterältesten, in den Wojwodschaften die Wojwoden. Die Gerichtssitzungen fanden viermal im Jahr statt. Hier erfolgten die Verhandlungen in Straf- und Zivilsachen der Schlachta (Adel) und der abgabenpflichtigen Stände des Großfürstentums Litauen. Sie wurden in den Gouvernements Witebsk, Minsk, Moghilew im Jahre 1795 und im Gouvernement Grodno im Jahre 1831 mit der Übergabe der Funktionen an die Kreisgerichte abgeschafft. Auf dem Territorium Weißrusslands existierten 35 solcher Stadt- und Burggerichte; 31 davon in weißrussischen Städten.

\section{c) Landgerichte}

Den Kreisgerichten von verwaltungsgerichtlichen Kreisen auf dem Territorium des Großfürstentums Litauen entsprechen die Landgerichte der Schlachta. Sie wurden im Großfürstentum Litauen 1565 gegründet. In Polen existierten sie seit dem 13. Jahrhundert. 
Die Landgerichte bestanden aus einem Richter, einem Unterrichter und einem Schreiber. Die Gerichtssitzungen fanden dreimal im Jahr statt. Sie verhandelten in Straf- und Zivilsachen und übten auch die Funktionen eines Notariats aus. Auf dem Territorium Weißrusslands existierten 25 solcher Landgerichte; 23 davon in den weißrussischen Städten.

\section{d) Kapturgerichte}

Die Kapturgerichte wurden während der Thronvakanzen im Jahre 1572 als provisorische Gerichte gegründet. Auf einem Sejm (Forum) wählte man auf Versammlungen (Konvokationen) die Kapturrichter, die in Straf- und Zivilsachen verhandelten. Diese Gerichte wurden im Jahre 1768 auf Beschluss des ordentlichen Sejms aufgelöst. Auf dem Territorium Weißrusslands existierten 15 solcher Kapturgerichte; alle in weißrussischen Städten.

e) Oberverwaltungsgerichte oder Podkomorskie - Gerichte für Angelegenheiten der Vermessung von Landgütern

Diese Gerichte wurden im Jahre 1566 nach dem Zweiten Statut des Großfürstentums Litauen gegründet. Nach dem Dritten Statut von 1588 wählte man in jedem Kreis (Powjat) vier Kandidaten, von denen der König einen als Oberlandmesser (Podkomorij - oder als Richter für Bodenstreitigkeiten) bestätigte. Der Letztgenannte zog einen oder zwei Unterlandmesser (Komornik) zur Unterstützung heran. Sie beschäftigten sich vor Ort mit der Verhandlung der aus den Stadt- und Landgerichten eingehenden Sachen für Bodenstreitigkeiten und trafen die Entscheidungen in Form einer Grenzliste. Die Appellationen legte man beim Kammergericht des Großfürstentums Litauen ein. Diese Gerichte wurden in Ostweißrussland im Jahre 1772, in West- und Zentralweißrussland im Jahre 1795 abgeschafft. Sie wurden im Jahre 1797 vom russischen Kaiser Paul I. wiedereingesetzt und am 11. Mai 1832 erneut aufgelöst. Auf dem Territorium Weißrusslands existierten 17 solcher Oberverwaltungsgerichte; alle in weißrussischen Städten.

\section{f) Kommissarische Gerichte}

Kommissarische Gerichte sind provisorische Gerichte, die zur Behandlung von Streitigkeiten zwischen der Verwaltung von königlichen Landgütern, Privatbesitzern und Magistraten vom König eingerichtet wurden. Die Kommissare behandelten die Sachen vor Ort mit Vorladung der Streitparteien und Zeugen. Über jede Verhandlung führte man ein Protokoll. Die Appellationen wurden beim Zadvorny Assessorgericht (Königlichen Gerichtshof) des Großfürstentums Litauen eingereicht. Auf dem Territorium Weißrusslands existierten fünf solcher Kommissarischen Gerichte; alle in weißrussischen Städten.

\section{g) Schiedsgerichte oder Kompromissarische Gerichte}

Schiedsgerichte oder Kompromissarische Gerichte sind Gerichte für die schiedsgerichtlichen Verhandlungen, die aus Richtern verschiedener Ränge und Stände bestanden. Deren Geschäftsordnung wird durch das Zweite Statut des Großfürstentums Litauen von 1566 bestimmt. Sie verhandelten in Straf- und Zivilsachen, mit Ausnahme von Sachen zwischen den Besitzern der königlichen Landgüter und von Strafsachen, die mit den Interessen des Ärars (Fiskus) verbunden waren. Die Appellationen legte man beim Kammergericht des Großfürstentums Litauen ein. Diese Gerichte wurden durch den Erlass von Zar 
Nikolaus I. am 25. Juni 1840 aufgehoben. Auf dem Territorium Weißrusslands existierten 14 solcher Schiedsgerichte; alle in weißrussischen Städten.

\section{Familienbestände und Bestände zur persönlichen Abstammung}

A) Bylgaki Bestand 3250, 55 Bündel, 1630-1915

B) Elenskie Bestand 1636, 206 Bündel, 1578-1908, F. 79

C) Khmary Bestand 1324, 1766 Bündel, 1508-1882

D) Lubomirscy Bestand 3258, 108 Bündel, 1578-1908, F. 79

E) Plater-Siebergs Bestand 1503, 398 Bündel, 1519-1875

F) Radziwills Bestand 694, 25585 Bündel, 1366-1937

\section{Sammlungen}

\section{a) Urkundensammlungen}

Die Urkundensammlungen enthalten topographische Karten und Pläne von Dörfern, Ortschaften und Landgütern des Gouvernements Minsk.

\section{b) Sammlungen der Mikrofilmkopien von Urkunden}

Die Sammlung Nr. 5 enthält Urkunden zur Geschichte Weißrusslands aus den Beständen der Fürsten Radziwill und Sangushki, der Grafen Pšezdezkij und Tisengaus, aus dem Hauptarchiv der Alten Akten in Polen, aus dem Staatsarchiv in Krakau, aus dem Archiv der Neuen Akten in Warschau, aus der Nationalbibliothek in Warschau, aus der Bibliothek des Ossolineums in Wrocław (Breslau); insgesamt 255.867 Dokumente aus den Jahren 1501-1935. Die Sammlung ist zwischen 1967-1995 nach Minsk gekommen.

Die Sammlung Nr. 9 besteht aus dem Findbuch des Bezirks (Starostwo) und der Stadt (der Burg) Moghilew aus der Bibliothek bei der Akademie der Wissenschaften von Litauen; 447 Aufnahmen aus dem Jahr 1560. Die Sammlung ist im Jahre 1960 nach Minsk gekommen.

Die Sammlung Nr. 18 enthält die Litauische Matrikel (Litauische Metrik) und das Archiv der Kanzlei des Großfürstentums Litauen aus dem Russischen Staatsarchiv für Alte Akten in Moskau; 448.074 Dokumente aus dem 15.-18. Jahrhundert. Die Sammlung ist im Jahre 1992 nach Minsk gekommen.

\section{II. Überblick über die Quellen des deutschen Rechts, die zur Zeit in der Nationalbibliothek Weißrusslands in Minsk aufbewahrt werden}

Die weißrussischen Ortschaften des Großfürstentums Litauen erhielten im Laufe ihrer Entwicklung und Umwandlung in Handels- und Handwerkszentren eine Ordnung nach deutschem Recht, nach dem Vorbild der westeuropäischen Länder. Als deutsches Recht bezeichnete man in den Privilegien am häufigsten das Magdeburger Recht, selten seine Varianten, z.B. das Kulmer Recht (jus Culmense - Stadt Chelmno im polnischen Preußen) und das Schrodzer Recht (jus Sredense v Novi Fori - Stadt Slenska Sroda (Neumarkt), im polnischen Schlesien).

Ein charakteristisches Merkmal des Kulmer Rechts bestand darin, dass es kleinen, insbesondere neu entstandenen und besiedelten Ortschaften erteilt wurde. 
Aufgrund der Suche nach den Quellen, die in direkter Beziehung zu den Fragen der Rezeption des Magdeburger Rechts in den weißrussischen Ländern des Großfürstentums Litauen stehen und die sich jetzt in der Nationalbibliothek Weißrusslands befinden, habe ich die folgende Übersicht verfasst:

\section{Quellen des deutschen Rechts in weißrussischen Ortschaften des Großfürstentums Litauen}

In weißrussischen Ortschaften des Großfürstentums Litauen waren die Privilegien des Souveräns oder des Stadtherrn die grundlegenden Akte, die die Statuten der Deutschrechtstädte bestimmten. Die Privilegien nach deutschem Recht kannten drei Hauptquellen des Stadtrechtes für Städte, in denen Folgendes eingeführt wurde:

a) Königliche bzw. großfürstliche Privilegien;

b) Willküren, Plebiscita der Stadtgemeinden;

c) Kodizes oder Sammlungen des Magdeburger bzw. Kulmer Stadtrechts. ${ }^{2}$

\section{a) Privilegien nach deutschem Recht}

Zur Hauptquelle des Magdeburger Rechts in Weißrussland gehören die Privilegien. Die Urkunden sind veröffentlicht in den »Urkunden des Westlichen Russlands«, »Urkunden des Südlichen und Westlichen Russlands«, »Historisch-juristische Materialien, die aus Aktenbücher in den Gouvernements Witebsk und Moghilew entnommen worden waren«, »Akten der Archäologischen Komission zu Wilna«, in der dreibändigen Quellensammlung »Archiv Weißrusslands« sowie in anderen russischen und polnischen Sammlungen.

Die Urkunden des Westlichen Russlands (AZR), die in der Nationalbibliothek Weißrusslands verwahrt werden, stellen eine fünfbändige Quellensammlung hauptsächlich für die politische Geschichte der Ukraine, Weißrusslands und Litauens im Zeitraum von 1340 bis 1699 dar. Die Bände zwei und drei dieser Sammlung enthalten die Texte der Hauptund Konfirmationsprivilegien nach dem Magdeburger Recht für die Städte Weißrusslands. Aufgrund von deren Analyse kann man für viele Städte die charakteristischen Privilegien sowie deren Besonderheiten erkennen. Die wirtschaftliche Entwicklung einzelner Städte bzw. ihre geographische Lage haben ihnen oft ein besonderes Gepräge gegeben. Die zweite Sammlung von Urkunden, die in der Nationalbibliothek von Minsk verwahrt wird, ist die fünfzehnbändige Quellensammlung »Urkunden des Südlichen und Westlichen Rußlands (AJZR)«. Hier sind die Dokumente für die Geschichte Russlands, der Ukraine, Weißrusslands und Litauens für den Zeitraum von 1361 bis 1678 versammelt. Die größte Anzahl von Angaben für die Geschichte der Städte Weißrusslands befindet sich im zweiten Band, dessen Quellenmaterial, vor allem zur Charakteristik von Privilegien nach Magdeburger Recht sowie zur Frage der Tätigkeit von Stadtverwaltungen Auskunft gibt. Hier kann man z.B. eine Schenkungsurkunde für die Einwohner der Ortschaft Woinski nach Magdeburger Recht vom 26.07.1511³ finden oder eine königliche Schenkungsurkunde für die Bürger

2 Die oben genannte Klassifikation wurde von dem ukrainischen Forscher Andrij Jakowliw in seiner Arbeit »Das deutsche Recht in Weißruthenien« in Umlauf gebracht, die in Berlin im Jahre 1944 erschien: JAKOWLIW, A. Das deutsche Recht in Weißruthenien. Berlin: Selbstverlag der Publikationsstelle, 1944, S. 22-23.

3 Archiv jugo-zapadnoj Rossii (Archiv des süd-westlichen Russlands; im Folgenden: AJZR). Bd. 1. Sankt-Petersburg, 1863, S. 80-81. 
von Minsk über die Durchführung zweier Messen und die Erhebung von Gebühren für über die Brücke des Flusses Swisloč transportierte Waren vom 10.02.1571.4

In der Nationalbibliothek besteht die Möglichkeit die dreißigbändige Urkundensammlung »Historisch-juristische Materialien, die aus den Aktenbüchern in den Vitebsker und Mogiljowsker Gouvernements entnommen wurden « (IJM) ein-zusehen. Mit den Lieferungen IV, V und VI liegen die Urkundenbücher der Stadtverwaltung Polozk aus den Jahren 1633 bis 1782 vor. ${ }^{5}$ Jede gerichtliche Festlegung weist einen Bezug zu den Artikeln aus »Spectrum Saxonicum« (Saxonicum, prawo Saxonow), »Jus Municipale« (Prawa Maydeburskiego) und aus der Kompilation »Porzadku Prawa Maydeburskiego« auf.

In der weißrussischen Abteilung der Nationalbibliothek liegt eine bekannte neun- und dreißigbändige Urkundensammlung »Akten der Archäographischen Kommission zu Wilna« (AWAK). Der Band I enthält die Akten des Landgerichts von Grodno seit dem Jahr 1540 bis 1795,6 Band II die Akten des Landgerichts von Brest für den Zeitraum 1431 bis 1793, ${ }^{7}$ Band III die Akten des Stadtgerichts von Brest seit dem Jahr 1401 bis 1699 usw.

\section{b) Betrachtung konkreter Beispiele}

aa) Erstes Beispiel aus der Lieferung VIII der »Historisch-Juristischen Materialien «

19. April 1624. Die gerichtliche Entscheidung über die Klage des Bürgers Fjodor Rostopa wegen Diebstahls.

Ein Bürger von Mogiljow beklagte sich durch seinen Bevollmächtigen Woitech Lapa über Fjodor Rostopa, Bürger von Mogiljow, weil dieser in sein Kämmerchen auf dem örtlichen Markt verbrecherisch und heimlich eingedrungen sei, dieses mit den gestohlenen Schlüsseln geöffnet habe sowie Geld in Höhe von dreißig litauischen Groschen und neunzehn Kopeken mitgenommen habe. ${ }^{8}$

Dieser Straffall nahm ursprünglich Bezug auf den zweiten Teil der »Ordnung«, der besagte, dass als Verbrechen eine Verheimlichung der gestohlenen Sache gilt. Darum geht es auch in Kapitel 47 des Magdeburger Rechts, Artikel 92, 114, 115 und 135, sowie in den Artikeln des »Königlichen Rechts«.

Dann nimmt das Gericht Bezug auf die Blätter 10 und 11 »Über gestohlene Sachen«, die man bei jemandem gefunden hat, oder über Instrumente, mit denen Türen und Schlösser geöffnet wurden; auf das Kapitel 124 des vierten Teils der »Ordnung«; auf das Kapitel 48 des zweiten Teils der »Ordnung«; auf das Magdeburger Recht, Artikel 36 und 55 des ersten Buchs; Artikel 66 und 70 des zweiten Buchs sowie Artikel 10 des zweiten Buchs; auf das Magdeburger Recht, Artikel 5 des dritten Buchs; im »Speculum Saxonum« im Kapitel 153; auf das Blatt 21 der »Eidordnung«; auf das Kapitel 20 der »Ordnung« über Kenntnisse, Argumente und Ablehnungsgründe usw.

In diesem Fall werden neben drei anderen die zwei wesentlichen deutsch-rechtlichen Quellen (Sachsenspiegel und Magdeburger Recht) verwendet.

$4 \quad$ AJZR 1, 1863, S. 180-181.

5 Aus den Aktenbüchern des Vitebsker und Mogiljowsker Gouvernements (im Folgenden: IJM). Lief. IV. Witebsk, 1873; IJM, Lief. V. Witebsk, 1874; IJM, Lief. VI. Witebsk, 1874.

6 Akten der Archäographischen Kommission zu Wilna (im Folgenden: AWAK). Bd. I. Wilna, 1865.

7 AWAK, Bd. II. Wilna, 1867 (insgesamt X, 361 S.).

8 IJM, Lief. VIII. Witebsk, 1877, S. 397-406. 
bb) Zweites Beispiel aus der Lieferung IV der "Historisch-Juristischen Materialien"

10. Juni 1652. Nach der gerichtlichen Entscheidung in der Sache des Bürgers Iwan Tischkovič und des Lotsen Daniil Konoval.

Anläßlich des Floßbruches mit Waren nach Riga auf dem Fluss Düna wurde am 10. Juni 1652 von beiden Parteien gegen einige Paragraphen dieser Entscheidung eine Kassationsklage vor dem Königsgericht eingelegt. ${ }^{9}$

Hier finden wir folgende Bezugnahmen auf»Speculum Saxonicum«: Blatt 579, Stichwort »Anvertrautes Gut«, in dem gesagt wird, wie man anvertrautes Gut behandeln muss; Blatt 260, Stichwort »Gebühr«, in dem es um die Verantwortlichkeit des Dieners geht und was wegen seiner Unvorsichtigkeit geschehen kann; Blatt 430, Stichwort »Über die Strafe des Dieners«, in dem der Schuldlose in vier Fällen zum Schuldigen wird; Blatt 270, Stichwort »Beschädigung«; Blatt 452, in dem es um Fahrlässigkeitsverfehlungen und deren Bestrafung geht und Blatt 330, in der Anlage zur Gerichtsordnung. Außer der Bezugnahme auf die oben genannten Regelungen war Iwan Tischkovič bereit, »selbviert « zu beschwören, dass diesen Verlust nur der Lotse Daniil Konoval verschuldet habe. ${ }^{10}$

Das bedeutet, dass in diesem Prozess materielles Recht (die Tatbestandsbegriffe) des »Sachsenspiegels« angewandt wurde. Am 19. Juni 1652 sagten als Zeugen die Arbeiter Waska Fedorovič und Fjodor Tiwunenka, die sich im Moment der Tragödie auf dem Floß befanden und auf die Forderung des Gerichtsvollziehers hin, persönlich vor dem Gericht erscheinen sollten, unter anderem, unter Anwendung des Artikels des Magdeburger Rechts mit nach oben erhobenen zwei Fingern aus. ${ }^{11}$ In der Prozessordnung (Verfahrensrecht) war das Magdeburger Recht maßgeblich.

\section{cc) Drittes Beispiel aus der Lieferung VI der »Historisch-Juristischen Materialien"}

\section{Februar 1683. Eine gerichtliche Entscheidung in der Sache Stefan Amwrossijevič} Kuksa und Mark Ivanovič Syssa anläßlich der Tötung seiner, Syssas, Ehefrau. ${ }^{12}$

Mark Syssa wurde auf Grund des Magdeburger Rechts wegen einer schweren gotteswidrigen Straftat, der Tötung seiner Ehefrau Poloneja Syssina, verurteilt, die von ihm unbarmherzig zu Tode gequält wurde. Hier wird Bezug auf Spectr. Saxonic, Blatt 157, über Bestrafung, auf Blatt 232, Teil III der Gerichtsordnung, über vorsätzliche Tötung und auf Blatt $70 »$ Prawa Cesarskiego« (das Römische Recht), genommen. Unter Berücksichtigung der Tatsache, dass die Ehefrau Marks schwanger war, trifft das Gericht die Entscheidung, Syssa durch Vierteilung hinzurichten. Am dritten Tage, d.h. am 11. Februar, wurde er aus dem Rathaus auf den Marktplatz herausgeführt und öffentlich unter Anwendung des Magdeburger Rechts exekutiert. Bemerkenswert ist hier, dass in diesem Prozess sogar drei unterschiedliche Rechtssysteme angewandt wurden.

\section{c) Schlussfolgerungen}

Dieses sind die allgemeinen Schlussfolgerungen, welche ich in Übereinstimmung mit der Analyse von Informationen aus den gerichtlichen Akten ermittelt habe:

\footnotetext{
9 IJM, Lief. IV. Witebsk, 1873, S. 405-407.

$10 \quad$ Ibidem, S. 424.

11 IJM, Lief. V. Witebsk, 1874, S. 255-256.

12 IJM, Lief. VI. Witebsk, 1874, S. 274-285.
} 
Die Rechtsbücher des Magdeburger Rechts der Rzeczpospolita ${ }^{13}$ teilen sich in zwei Gruppen auf: 1) die echten Rechtsbücher: Landrecht (Speculum Saxonum) und Weichbild (Jus Municipale), die in Polen seit langem in verschiedenen deutschen und lateinischen Listen und seit 1535 ausschließlich im Text von Jasker vorkommen; 2) die örtlichen Rechtsbücher: das sind die Werke von Tserasin, Joannis Cervi Tucholiensisa, Bartholomäus Groicki und Pawel Szczerbicz. ${ }^{14}$ Welche dieser Rechtsbücher, die echten oder die örtlichen, wurden nun in den weißrussischen Städten mit Magdeburger Recht (=Magdeburgien) der Rzeczpospolita verwendet?

Meine Analyse einer ganzen Reihe von Gerichtsakten verschiedener Magdeburgien zeigt, dass man bei den Gerichten der weißrussischen Städte der Rzeczpospolita eindeutig nicht die echten, sondern die örtlichen (polnischen) Bücher des Magdeburger Rechts, d.h. die Werke von Bartholomäus Groicki und Pawel Szczerbicz, gebrauchte. Sie waren die einzigen Rechtsbücher des Magdeburger Rechts in den weißrussischen Städten der Rzeczpospolita.

Man muss zugleich erwähnen, dass es diese Formen des Magdeburger Rechts in den polnischen Originalen der Bücher von Groicki und Szczerbicz waren, die bei den Gerichten weißrussischer Magdeburgien Gültigkeit hatten. Einige dieser Bücher wurden ins Russische übersetzt, aber die Übersetzungen waren sehr selten und vor allem sehr unvollkommen. »Die Mehrheit der russischen Übersetzungen«, teilt der hervorragende Forscher F. W. Taranovski Ende des 19. Jahrhunderts in seinem klassischen Werk mit, »entstanden nach der Beendigung der polnischen Herrschaft infolge des Nachlassens der polnischen Sprachkenntnisse ${ }^{15}$

Die gerichtlichen Akten vieler Magdeburgien (zum Beispiel in Brest, Kobrin ${ }^{16}$ ) enthalten nicht nur Hinweise auf diese oder jene Seite der Werke von Pawel Szczerbicz und Bartholomäus Groicki, sondern auch ganz wörtliche Auszüge aus diesen Werken. Ähnliche Hinweise und Auszüge werden nicht nur in den Gerichtsverfahren über die Durchführung von Exekutionen, sondern auch in einer ganzen Reihe von Straf- und Zivilsachen benutzt.

Auch ist interessant, dass in den gerichtlichen Akten, die in polnischer Sprache geschrieben sind, die Auszüge von Groicki und Szczerbicz im Original angeführt werden; aber in den Akten, die auf Russisch verfasst sind, bestimmte Fachausdrücke übersetzt werden.

Weiterhin ist es auch nicht unbedeutend, dass die Hinweise auf die Werke von Groicki und Szczerbicz am häufigsten nicht vom Gericht, sondern von den Beteiligten in die gerichtlichen Diskussionen gemacht werden. Die Diskussionen führen meistens nicht die Beteiligten selbst, sondern ihre Anwälte (die Prokuratoren). »Die historisch-juristischen Materialien« enthalten Informationen über das damalige Vorhandensein von Anwälten in den weißrussischen Städten der Rzeczpospolita. Als Beispiel sind zu nennen: Christophor

\footnotetext{
13 Rzeczpospolita war eine Föderation des polnischen Königreichs und des Großfürstentums Litauen. Sie wurde infolge des Abschlusses der Lubliner Union im Jahre 1569 gebildet.

14 TARANOVSKI, F. V. Übersicht über die Denkmäler des Magdeburger Rechts der westrussischen Städte der litauischen Epoche. II. Warschau, 1897, S. 33.

15 Ibidem, S. 51.

16 AWAK, Bd VI. Wilna, 1872, S. 593.
} 
Kozarin, dessen Tätigkeit in der Stadt Polozk in die Zeit von 1652 bis 1683 fällt, sowie seine Zeitgenossen, die Anwälte Michail Shochovski und Alexander Spakovitsch. ${ }^{17}$

In den Akten, die mit Hilfe von Anwälten geführt wurden, wurde besonders oft Bezug auf die Bücher von Szczerbicz und Groicki genommen. Hieraus entsteht der Eindruck, dass diese Berufsanwälte, wenn sie auch nicht professionelle Juristen waren, sich wenigstens auf dem Gebiet der polnischen Literatur des Magdeburger Rechts auskannten. Ebenso hatten die Anwälte einen sehr wesentlichen Einfluss auf die Verbreitung der Werke von Groicki und Szczerbicz in den weißrussischen Städten der Rzeczpospolita. Nur mit ihrem Einfluss ist die Tatsache der schnellen Rezeption der polnischen Bücher des Magdeburger Rechts durch die Bürger von weißrussischen Magdeburgien der Rzeczpospolita zu erklären. Als Beispiel kann man die Stadt Moghilew anführen, die das Magdeburger Recht im Jahre 1577 bekam. Dort wurde im Jahre 1585 in einer Gerichtssache, genauer gesagt in einer Diebstahlsache, eine ganze Reihe der Auszüge aus der »Ordnung « von Bartholomäus Groicki angeführt, die sowohl die Beteiligten des Verfahrens, als auch das Gericht verwendeten. ${ }^{18}$ Nach Meinung von F. W. Taranovski wurde der Titel »Ordnung« als Oberbegriff für alle gesammelten Werke von Groicki verwendet. ${ }^{19}$

So kann man behaupten, dass sich im 16. und 17. Jahrhundert in der Rzeczpospolita ein erhöhtes Interesse an der Jurisprudenz beobachten lässt. Einige Juristen (Bartholomäus Groicki, Pawel Szczerbicz u.a.) unterstützten die Verbreitung des Rechts mit ihrer literarischen Tätigkeit; andere Juristen (Christophor Kozarin, Alexander Spakovitsch, Michail Shokhovski u.a.) verwirklichten durch ihre anwaltliche Praxis die Arbeiten der Ersteren. ${ }^{20}$

Es ist wichtig, noch einen wesentlichen Aspekt anzuführen: zwar wurde das Magdeburger Recht in den weißrussischen Städten der Rzeczpospolita als angewandtes Recht genutzt; in der gerichtlichen Praxis wurde es in Gestalt der polnischen Darlegungen von Groicki und Szczerbicz verwendet, neben dem Magdeburger Recht aber galt in den Gerichten weiterhin auch das örtliche Gewohnheitsrecht. ${ }^{21}$

\section{Die städtischen und gerichtlichen Statuten, Willküren, Plebiscita}

Die städtischen Beschlüsse können je nach Inhalt und Art ihrer Beschlussfassung in zwei Gruppen eingeteilt werden: a) Die Verordnungen, die auf eine Umgestaltung der Stadtverwaltung oder der Gerichtsordnung abzielten und b) Die Verordnungen über die Einführung neuer Steuern, über außerordentliche Ausgaben aus städtischen Mitteln, über die Entsendung von städtischen Abgeordneten an den Reichstag oder den König usw.

17 SOZONOV (Hg.). Historisch-juristische Urkunden. Die aus den Büchern der Stadtverwaltung Polozk entzogenen Akten. Bd. IV. Vitebsk, 1873; ders. Historisch-juristische Urkunden. Aktenauszüge aus den Büchern der Stadtverwaltung Polozk. Bd. V. Vitebsk, 1874; ders. Historisch-juristische Urkunden. Aktenauszüge aus den Büchern der Stadtverwaltung Polozk. Bd. VI. Vitebsk, 1874.

18 Ders. Historisch-juristische Urkunden. Aktenauszüge aus den Büchern der Stadtverwaltung Polozk. Bd. VII. Vitebsk, 1876, S. 369-372.

19 TARANOVSKI, op. cit., S.190.

20 Ibidem, S. 47.

21 Ibidem, S. 55. 


\section{a) Die Verordnungen, die auf eine Umgestaltung der Stadtverwaltung oder}

der Gerichtsordnung abzielten

Als Beispiel einer Verordnung der ersten Art sei die Willkür der Stadt Moghilew über die Organisation eines Vertreterkollegiums aus zwölf Personen angeführt: »Während der 12 Jahre seit der Verleihung des Magdeburger Rechtes an die Stadt Moghilew (1577) haben die städtischen Bürgermeister keine Rechenschaft über die städtischen Einnahmen und Ausgaben vor der Stadtgemeinde abgelegt, überdies haben sie sich bei der Wahl von Ratsherren und Bürgermeistern verschiedener Missbräuche schuldig gemacht. Infolgedessen hat die Stadtgemeinde, an diesem 13. Oktober 1588 an einem Ratstag auf dem Rathaus versammelt, einstimmig erkannt und beschlossen: Zur Verwaltung und Verfügung über die städtischen Einnahmen und verschiedene städtische Genüsse, ebenso zur Wahl des Stadtrates, der Bürgermeister, Ratsherren und Schöffen gemäß dem Privileg ebenso wie zur Entgegennahme der Abrechnungen vom Stadtrat, aus unserer Mitte 12 Bevollmächtigte zu wählen, von denen je 4 Personen jedes Jahr über die städtischen Einnahmen zu verfügen haben, immerdar. Diesen 12 gewählten Vertretern erteilt die Stadtgemeinde eine ausreichende Vollmacht sowohl für die Wahl des Stadtrates - der Herren Bürgermeister, Räte und Schöffen - gemeinsam mit dem vorhergehenden Stadtrat - aus Personen ihres Kreises, die von ihnen als stadtnützlich und rechtskundig befunden werden, als auch für die Verwaltung der Einnahmen und verschiedener städtischer Nutznießungen, auf dass die städtischen Genüsse sich mehren und ausbreiten und unsere Brüderschaft keinen Nachteil erleide. Und wo die städtischen Einnahmen zur Deckung der städtischen Bedürfnisse nicht langen, erteilen wir ihnen einmütig das Recht, gemeinsam mit dem Stadtrat zu beschließen, und wir werden für die städtischen Bedürfnisse ohne jegliche Weigerung beisteuern, immerdar. Diese Beschlüsse müssen mit dem Stadtsiegel sowie mit den persönlichen Siegeln und eigenhändigen Unterschriften der gewählten Vertreter versehen sein, ohne welches der Beschluss ungültig ist. Die städtischen Gelder sollen auf Grund dieser Beschlüsse städtische Schaffner einsammeln, die je zwei und zwei vom Stadtrat und vom Vertreterkollegium gewählt werden müssen; nach Ablauf eines Jahres haben die Schaffner vor dem Stadtrat und dem Vertreterkollegium Rechenschaft abzulegen und Quittungen zu erhalten. Sollte jemand von den 12 Männern aus triftigen Gründen abwesend sein, so besitzen die restlichen 10, 8, 6 und selbst 4 von den Anwesenden die Vollmacht, für alle 12 und für die ganze Gemeinde zu handeln. Sollte sich aber jemand von den 12 unordentlich zeigen oder einen anderen Grund geben, so wird er von der Gemeinde beseitigt und ein anderer an seine Stelle gewählt, ebenso wenn jemand aus triftigen Ursachen auf sein Amt verzichtet, ist ein anderes Mitglied zu wählen«.

Die Stadtgemeinde, bestehend aus zwölf Hundertschaftsmännern mit ihren Zehntmännern und allen Gemeindemitgliedern, »die wegen ihrer großen Zahl nicht aufgezählt noch namentlich aufgeschrieben werden können: es waren eben alt und jung«, erschien vor dem Landvogt, den Bürgermeistern, Ratsherren und Schöffen, vor dem ganzen Rat, dem neuen wie dem alten, verlautbarte einmütig diesen Beschluss und legte ihn zur Bestätigung vor. Die Bürgermeister, Ratsherren und Schöffen haben, nach gepflogener Beratung, diesen Beschluss einstimmig bestätigt und dem Schreiber befohlen, denselben in die städtischen Gerichtsbücher einzutragen. Ein Auszug aus den Büchern wurde hierauf dem König 
Sigismund III. zur Bestätigung unterbreitet und dieser bestätigte ihn auch mit dem Privileg vom 1. Februar $1588 .^{22}$

b) Die Verordnungen über die Einführung neuer Steuern, über außerordentliche Ausgaben aus städtischen Mitteln, über die Entsendung von städtischen Abgeordneten an den Reichstag oder den König usw.

Als Beispiel einer Willkür der zweiten Art kann der Beschluss der Moghilewer Bürgerschaft vom 3. April 1625 über die Steuererhöhung zur Deckung der städtischen Ausgaben dienen: »Auf dem Rathaus versammelt, haben wir, die Bürgermeister, Ratsherren und Schöffen des neuen wie des alten Rates gemeinsam mit den 12 Männern, die von der bürgerlichen Gemeinde gewählt wurden, indem wir für die ganze Stadt dringend Geld benötigen und diesen Bedarf aus den städtischen Einnahmen nicht decken können, beschlossen: von jeder Rute Siedlungsland je 2 Dreier und von jeder eingezogenen einen Dreier zu nehmen; von Weideplätzen und Äckern 2 Dreier pro Morgen und von Moorwiesen einen Dreier; von Kaufläden je einen polnischen Gulden, von Waaghäusern und kleineren Läden je 12 Groschen, von Ellen 2 Dreier, von städtischen Schlachthäusern 6 Schock litauische Groschen. Die beschlossenen Gelder sind von den jährlichen Hundertschaftsmännern einzuheben und den städtischen Schaffnern abzuliefern. Zum Gedenken in die Moghilewer städtischen Bücher eingetragen«. ${ }^{23}$

\section{Die Gesetzbïcher und Sammlungen des Magdeburger und Kulmer Rechts}

Bekanntlich wurde das »Magdeburger Weichbild« zu einer der nichtauthentischen Quellen des Magdeburger Rechts. Dieses wurde auf Grundlage des »Sachsenspiegels« entwickelt. Gerade in diesem Zusammenhang liegen allen Übersetzungen und Fassungen des Magdeburger Rechts die Bestimmungen aus dem »Sachsenspiegel « und dem »Magdeburger Weichbild« zugrunde, was man in den Arbeiten von J. Łaski, M. Jaskier, I. Terasin, B. Groicki, P. Szczerbicz u.a. erkennen kann.

Die Nationalbibliothek der Republik Belarus besitzt ein Buch mit dem Titel »Speculum Saxonum«. Dieses Buch befindet sich in der Abteilung der seltenen Bücher und hat die Signatur Nr. 09 allg. / 3534. Das Buch enthält zwei bei den weißrussischen Gerichten dieser Zeit gut bekannte Werke des polnischen königlichen Sekretärs Pawel Szczerbicz »Speculum Saxonum albo Prawo Saskie y Maydeburskie« und »Jus Municipale to jest Prawo Miejskie Maydeburskie«, die im Jahre 1581 verlegt wurden. Die Bibliothek hat leider keine späteren Ausgaben von 1617 und 1646.

Der ukrainische Forscher Andrej Jakowliw wies schon im Jahre 1944 in seiner Untersuchung anlässlich der Rezeption des Magdeburger Rechtes in den Ortschaften Weißrusslands auf Folgendes hin: »Michal Balinski bringt in ,Starozytna Polska“" III, 627-628, den kurzen Inhalt des Privilegs, das Fürst Nikolaus Christoph Radziwill 1586 seiner Residenzstadt Neswisch auf Grund eines königlichen Privilegs aus dem gleichen Jahre verlieh. In diesem Privileg wird ausdrücklich verfügt, dass die Stadt Neswisch für den Gebrauch des ihr verliehenen Magdeburger Rechts das erstmalig im Jahre 1581 erschienene, Speculum

22 IJM, Lief. XIV. S. 438-444.

23 IJM, Lief. XXX. Vorwort, S. X. 
Saxonum“ von Paul Szczerbicz zu benutzen habe«. ${ }^{24}$ Das Buch, das jetzt in der Abteilung seltener Bücher bei der Nationalbibliothek Minsk aufbewahrt wird, ist aus der Bibliothek des Ordinariats Neswisch geliefert worden. Es regt zum Nachdenken an, dass man im Gericht der Residenzstadt Radziwills Neswisch gerade dieses Buch benutzte!

Es ist daran zu erinnern, dass man die Arbeiten von Pawel Szczerbicz in den gerichtlichen Akten wie folgt zitierte: »Speculum Saxonum«, dann folgte ein Schlüsselwort, zum Beispiel: »König«, weiter ein Artikel unter bestimmter Nummer und bestimmter Paragraf. Pawel Szczerbicz selbst hat die Artikel in Paragrafen eingeteilt; bei seinem Vorgänger Jaskier kann man das noch nicht beobachten. Beide Arbeiten wurden in polnischer Sprache, in der Übersetzung aus den lateinischen und deutschen Texten, zur praktischen Anwendung für die Stadtverwaltungen und die einfachen Leute geschrieben.

Die Arbeit »Speculum Saxonum albo Prawo Saskie y Maydeburskie, porzadkiem abiecadla, z Lacinskich y Niemickich exemplarzow zebrane: a na Polski iezyk z pilnoscia y wiernie przez Pawla Szczerbicza na ten czas Sekretarza J. K. M.« ist alphabetisch geordnet. In den kleinen Kommentaren wird der Inhalt des »Sachsenspiegels« und des »Magdeburger Weichbildes« mit den Glossen zu verschiedenen Themen (z.B. Bestrafung, Eidschwur, usw.) angegeben. Als Muster zur Form dieser Sammlung diente das »Promptuarium« von Jaskier. Dasselbe »Promptuarium« benutze Pawel Szczerbicz auch bei der Auswahl einzelner Bestimmungen im »Speculum Saxonum« und im »Jus Municipale«, um sie in die einzelnen Rubriken seiner Sammlung einzuordnen. Dabei schöpfte er nicht das ganze Material des Promptuariums aus. Bei der Erstellung Originaltextes der sächsischen Rechtsdenkmäler wurden von Pawel Szczerbicz der lateinische Text von Jaskier sowie die deutschen Verzeichnisse benutzt.

Das »Jus Municipale To iest Prawo Mieyskie Maydeburskie nowo z Lacinskiego y z Niemieckiego na Polski iezyk z pilnoscia y wiernie przelozone Przez Pawla Szczerbicza Sekretarza J. K. M.« enthält ausführlich alle 140 Artikel des »Magdeburger Weichbildes« mit den Glossen und Kommentaren von Pawel Szczerbicz. Vor jedem Artikel befindet sich ein Verzeichnis seiner Hauptbestimmungen (Summa). Die Summa des ersten Rechtsartikels ist die größte. Sie besteht aus den 35 Hauptbestimmungen, unter denen man z.B. die folgenden nennen kann: das Recht und die Rechtsarten (sächsisches, römisches u.a.); was ist ein Recht und wo sind seine Urquellen; was ist Gerechtigkeit usw.

Die Praxis der Magistratsgerichte spricht dafür, dass sich die Parteien beim Gerichtsprozess zur Bestätigung ihrer Rechte vielmehr auf die Arbeiten von Groicki als auf die Arbeiten von Szczerbicz berufen. Auch die Arbeiten von Groicki sind in der Nationalbibliothek der Republik Belarus (Minsk) einsehbar.

Die Arbeiten von Groicki: a) »Artykuly«, b) »Porzadek«, c) »Postepek«, d) »Tytuly« sind vor allem dadurch interessant, dass man sie als eine Bearbeitung des Magdeburger Rechts unter dem Einfluss der Ideen der Renaissance und der neuen Rechtsdoktrin des 16. Jahrhunderts versteht.

24 JAKOWLIW, op. cit., S. 31. 
a) »Artykuly prawa majdeburskiego, ktore zowia Speculum Saxonum. Z Lacinskiego Jezyka na Polski przelozone «25 (= Artikel des Magdeburger Rechts, die man Speculum Saxonum nennt)

»Artykuly« (1568) ist das Werk von Bartholomäus Groicki und gleichzeitig die erste Fassung des Magdeburger Rechts in polnischer Sprache. Die »Artykuly« (,Artikel“) halten sich enger an die Texte der deutschen Quellen - den »Sachsenspiegel« und das »Magdeburger Weichbild«. Ein kleinerer Teil der Artikel hat als seine Urquelle das polnische Recht, nämlich das Gewohnheitsrecht von polnischen Magdeburgien sowie geschriebenes Statuten- und Sejmrecht. Ein besonderer Akzent wurde auf das Gebiet des Privatrechts gelegt und zwar auf Verpflichtungen aufgrund der Erbschaft, der Mitgift, der Realkaution der Witwe und des Testaments. Zum ersten Mal wird das neue Rechtsprinzip der gleichen Rechte des Mannes und der Frau auf Erbschaft begründet. Die »Artykuly« beinhalten eine Reihe von Rechtsinstituten, die in den Statuten fehlten. Dazu gehört z.B. die genealogische Tabelle zur Aufklärung der Erbschaftsrechte. Grundlage dieser Tabelle sei, so Groicki, der »Sachsenspiegel«, jedoch entwickelt er diesen weiter. Die Benutzung dieser Tabelle im in der Rechtspraxis war notwendig, da keines der Statuten diese enthält. Die Analogien dieser Arbeit schlossen nicht die möglichen Divergenzen in der Auslegung einiger Fachbegriffe aus. Als Beispiel muss man die außerehelichen Kinder erwähnen. Die »Artykuly« begründen die Legitimation solcher Kinder und die Anerkennung dieser als ehelich mit der Eheschließung der Eltern. In diesem Fall hatten die Kinder einen Anspruch auf Erbschaft von den Eltern. Ganz anders werden derartige Fälle im Statut geregelt. Die außerehelich geborenen Kinder gelten immer als unehelich, selbst wenn ihre Eltern später die Ehe geschlossen hatten. Sie haben demgemäß überhaupt keine Erbschaftsrechte. Doch noch mehr: Auch die von denselben (unverheirateten) Eltern geborenen Kinder, die schon in eheanbahnenden / ehelichen Beziehungen stehen, haben keine Erbrechte (III, Art. 28).

\section{b) »Porzadek sadow i spraw miejskich prawa majdeburskiego w Koronie Polskiej «26 (= Stadtgerichts- und Prozessordnung des Magdeburger Rechts im Kronland Polen $)^{27}$}

Die Arbeit von Groicki »Porzadek« (1559) enthält Normen des Prozessrechts, aber in den Normen der Prozessordnung sind organisch auch die Elemente des materiellen Rechts eingeschlossen, was typisch für damaliges Recht war. Das Hauptaugenmerk wird in dieser Arbeit auf die Annahme der Erbschaft, die Ausfertigung von Dokumenten und die gesetzliche Erbfolge gerichtet. Hier werden die Gründe angeführt, nach denen die Kinder ihr Erbrecht verlieren. Eine ähnliche Norm ist in den Statuten vorhanden, aber sie befindet sich in den verschiedenen Abschnitten und Artikeln (Statut 1588, V., Art. 8; Statut 1588, II. Art. 11). In den Statuten kann man die Normen finden, die für grundlegende

25 KORANYI, K. u. a. (Hg.). Bartolomiej Groicki: Artykuly prawa majdeburskiego. Warszawa: Wydaw. Prawnicze, 1954 (= ND der Ausgabe Krakow, 1565).

26 KORANYI, K. u. a. (Hg.). Bartolomiej Groicki: Porzadek sadow i spraw miejskich prawa majdeburskiego w Koronie Polskiej. Warszawa: Wydawnictwo Prawnicze, 1953 (= ND der Ausgabe Kraków, 1559).

27 In der Ukraine ist das Rechtsbuch unter der Bezeichnung »Porjadok praw ziwilnich magdeburskich (= Ordnung der Magdeburger Zivilrechte)« bekannt geworden. Zitiert nach: LÜCK, H. Magdeburger Recht in der Ukraine. ZNR, 1990, 12, S. 113-126, hier S. 122. 
Rechtsinstitute in den Arbeiten von Groicki charakteristisch sind. Zum Beispiel enthalten das Statut von 1588 und »Porzadek« adäquate Normen über den Ehebruch, der als Verbrechen gegen die Gesellschafts- und Familiensittlichkeit gilt (Statut 1588, XIV., Art. 29). Groicki jedoch gibt die Normen dieses Verbrechens ausführlicher an, weshalb man sich in den Akten von Magistratsgerichten gerade auf seine Arbeit beruft. Die Urquellen der Gerichtsordnung (Porzadek) von Groicki sind die folgenden:

1) das Magdeburger Recht, das in den glossierten »Speculum Saxonum« und »Jus Municipale« dargelegt ist, die der Verfasser für Porzadek aus zweiter Hand über »Farrago actionum« des Joannes Cervus Tucholiensis benutzte und die er auch in seinem »Porzadek« selbstständig übersetzte;

2) das Kaiserrecht, das als eine Hilfsquelle in »Porzadek« durch die Vermittlung von »Farrago actionum« und die »Praxis rerum criminalium« des Jodocus Damhouderius, eines berühmten holländischen Kriminalisten, einbezogen wurde;

3) das Gewohnheitsrecht der polnischen Magdeburgien;

4) das polnische, geschriebene Recht, die Königsstatuten.

c) »Ten postepek wybran iest s Praw Cesarskich, ktory Karolus V Cesarz kazal wydac po wszystkich swoich panstwiech, ktorym sye Nauka daie, iako w tych Sadziech a sprawach okolo karania na gardle, albo na zdrowiu, Sedziowie y kozdy Urzad ma sye zachowac, y postepowac wedle boiazni Bozey, sprawiedliwie, poboznie, rostopnie y nieskwapliwie ${ }^{28}$ (= Halsgerichtsverfahren)

Im »Postepek« (1559) wird von Groicki hauptsächlich der Text des deutschen Strafgesetzbuches von Kaiser Karl V. wiedergegeben, das unter dem Titel »Carolina« (die Peinliche Halsgerichtsordnung Kaiser Karls V., Constitutio Criminalis Carolina) bekannt ist. Forscher betonen die Tatsache der Anwendung und den Einfluss dieser Quelle nicht nur auf die Magistratsgerichte, sondern auch auf das gesamte Recht des Großfürstentums Litauen.

\section{d) »Tytuly Prawa Maideburskiego, do Porzadku y do Artykulow pierwey} po Polsku wyanych, W sprawach tego nawiecey klopotnych, s tegoz Prawa Maydeburs. Przydane ${ }^{29}$ (= Titel des Magdeburger Rechts)

Die letzte Arbeit »Tytuly« (1567) von Groicki, in der einige Rechtsnormen eingehender dargelegt und sogar verändert werden, ergänzt die vorhergehenden Werke. »Tytuly« enthält schon nicht mehr eine buchstabenmäßige Auslegung des »Sachsenspiegels«, sondern eine freie Interpretation und eigentlich eine dogmatische Wiedergabe Groickis unter Bezugnahme auf den »Sachsenspiegel«.

Was die unmittelbaren Quellen betrifft, aus denen Groicki das Rechtsmaterial für »Tytuly« bezog, so schloss er das Gewohnheitsrecht der Krakauer Magdeburgie aus der unmittelbaren Bekanntschaft mit der Krakauer Gerichtspraxis. Man kann mit vollem Recht behaupten, dass es, nach den verschiedenen Verfahren gegliedert, zum ersten Mal von Groicki aufgeschrieben wurde. Das Ziel, zu dem er, nach einigen Anfragen, die Normen des Gewohnheitsrechts der Krakauer Magdeburgie dargelegt hatte, wird in »Tytuly« nicht

28 Bartolomiej Groicki: Postepek sadow okolo Karania na gardle (Krakow, 1559). In: KORANYI, u. a. (Hg.), Bartolomiej Groicki: Artykuly prawa majdeburskiego.

29 KORANYI, K. u. a. (Hg.). Bartolomiej Groicki: Tytuly prawa majdeburskiego. Warszawa: Wydawn. Prawnicze, 1954 (= ND der Ausgabe Krakow 1557). 
genannt. Dafür sei aber der Blick auf eine Bestimmung zu lenken, die im Anhang zur Gerichtsordnung (»Porzadek«) aufgeführt ist: das Gewohnheitsrecht der Krakauer Magdeburgie wird nicht wegen seines materiellen Inhalts als obligatorisches Recht, sondern als formales Muster als nachahmenswert für andere Magdeburgien angeführt. Das Magdeburger und das römische Recht werden von Groicki nach den folgenden Quellen dargelegt: »Speculum Saxonum « und »Jus Municipale«, »Encheiridion aliquot locorum communium iuris Maideburgensis Joannis Kirsteyn Cerasini«, »Farrago actionum Cervi Tucholiensis«, $»$ Figura seu tabula indicans graphice quomodo haereditas ab intes-tato deferatur Stanislai Eichleri«, »Practica nova nunc temporis famosissima Joannis Petri de Ferrariis« und »Praxis rerum criminalium Jodoci Damhouderii«. Außerdem werden in »Tytuly« von Groicki selbst die Zitate aus dem »Corpus iuris civilis« aufgeführt, was in seinen anderen Werken fehlt.

\section{Das Nationalmuseum in der Stadt Neswisch}

\section{Allgemeines}

Im Jahr 1586 erhielt die Stadt Neswisch das Magdeburger Recht. Seit dieser Zeit folgte die Bebauung der Stadt einem regelmäßigen Plan. Den zentralen Teil der Stadt nahm eine große Fläche mit dem Rathaus und den Marktständen ein.

Das Rathaus von Neswisch ist ein baukünstlerisches Denkmal des 16. Jahrhunderts. Es ist das älteste unter den erhaltenen Rathausgebäuden auf dem Territorium Weißrusslands und wurde nach den Plänen Giovanni Maria Bernardonis, eines Baumeisters aus Italien, begonnen. Im sogenannten Kiewer Album von Bernardoni ist ein Blatt mit dem Plan der Rathausfundamente vom Ende des 16. Jahrhunderts - Anfang des 17. Jahrhunderts erhalten.

Das Privileg nach Magdeburger Recht wurde für Neswisch am 23. April 1586 in Grodno von Stephan Bathory, König von Polen (Rzeczpospolita) und Großfürst von Litauen, unterschrieben. Das gab der Stadt eine vollberechtigte Selbstverwaltung, Steuerbegünstigungen, eine Gerichtsimmunität sowie vorteilhafte Bedingungen für die Arbeit von Handwerkern und Händlern. Gleichzeitig erhielt Neswisch auch sein Wappen. Das Vorhandensein dieses Attributs sprach für ein ziemlich hohes wirtschaftliches und kulturelles Niveau, das die Stadt erreicht hatte. Das Wappen befand sich auch auf dem Stadtsiegel, das die Unabhängigkeit der Stadt Neswisch von der fürstlichen oder königlichen Macht betonte. Das zweite Privileg vom 18. Juni desselben Jahres wurde der Stadt durch den Besitzer von Neswisch, Nikolai Christophor Radziwill Sirotka, geschenkt. In Übereinstimmung mit dem letzten Privileg war die Stadtverwaltung verpflichtet, ein steinernes Rathaus zu bauen. Zehn Jahre später nach Erhalt des Magdeburger Rechts, 1596, wurde das Rathaus gebaut. Die erste bildliche Darstellung des Rathauses von Neswisch ist auf dem Stich von Tomasz Makowski (ca. 1605) überliefert. Der altertümliche Stich zeugt davon, dass auf dem Turm die Stadtuhr, die Glocken und eine Aussichtsplattform untergebracht waren.

In dem Gebäude des Magistrats, erbaut mit Mitteln und Kräften der Stadtbürgerschaft, befand sich während mehrerer Jahrhunderte für jedes Mitglied der Stadtgemeinde eine Hauptinstanz zur Lösung lebenswichtiger Probleme. Das Gebäude des Rathauses errichtete man in einem Ensemble mit den Marktständen, die aus zahlreichen Läden in Form 
von Zellen bestanden. Im 17. Jahrhundert zählte man bis zu 52 solcher Buden. Zwischen den Marktständen und dem Gebäude des Rathauses waren früher die Durchfahrten. In der sogenannten »Magdeburger Periode« (vom Ende des 16. Jahrhunderts bis zum Jahre 1786) befanden sich im Rathaus von Neswisch Kaufläden, ein städtischer Waagraum, eine Wachstube, eine Waffenhalle für die Bürger im Falle einer Gefahr, ein besonderer Raum zur Lagerung der Brandschutzeinrichtungen im Erdgeschoß und ein Gefängnis in den Kellerräumen. Der erste Stock war für die Magistratsbehörde bestimmt. Hier waren eine Ratsstube, ein Gerichtssaal, ein Verhandlungssaal, ein Ratssaal, eine Kanzlei, ein Schatzamt sowie die Vogt- und Bürgermeisterräume untergebracht. Hier befand sich auch das Stadtarchiv mit den Verwaltungsbüchern und Privilegien, die von den Königen und den Fürsten Radziwill der Stadt gegeben worden waren.

Das Rathaus ist im Nordischen Krieg beschädigt und im Jahre 1752 restauriert worden. Im Jahr 1836 wurde das Gebäude durch eine Feuersbrunst in Neswisch schwer beschädigt. Der Turm wurde von sechs auf vier Etagen verkleinert; die Räume im ersten Stock wurden beschädigt. Ungefähr seit 1849 befand sich im Rathaus eine orthodoxe Kirche; auch war einmal der Umbau des Magistratsgebäudes in eine orthodoxe Kathedrale geplant. Seit dieser Zeit und bis Ende des 19. Jahrhunderts wurden die Räume des Rathauses in den meisten Fällen nicht bestimmungsgemäß genutzt. Seit Ende des 19. Jahrhunderts und bis zum Jahr 1939 hatten im Gebäude wiederum die Verwaltungsorgane - das städtische Rathaus und die Stadtduma, das Kreisamt der Ältesten, die Polizei- und die Stadtverwaltung ihren Platz. Nach dem Zweiten Weltkrieg befanden sich hier das Kulturhaus, später das Pionier- und Schülerhaus sowie eine Kinderbibliothek.

Von 1997 bis 2004 wurden an dem baukünstlerischen Denkmal des 16. bis 18. Jahrhunderts, dem Rathaus von Neswisch, Restaurierungsarbeiten vorgenommen. Die Fassade des Gebäudes erhielt ihr ursprüngliches Aussehen zurück; die oberen Etagen des Turms, die Stadtuhr und die kleine Laterne mit Wetterfahne wurden restauriert, die Innenräume im ersten Stock für eine Museumsausstellung »Selbstverwaltung in der Stadt Neswisch« rekonstruiert. Diese Museumsausstellung informiert über die Hauptfunktionen der Selbstverwaltungsorgane im Neswisch zwischen dem 18. Jahrhundert und der ersten Hälfte des 19. Jahrhunderts sowie über deren Bedeutung im Leben der Stadtbewohner.

Es ist unter den Rathausgebäuden in Weißrussland heute die einzige Einrichtung, in der man die Tätigkeit des Stadtmagistrats kennenlernen kann.

\section{Der Ratssaal im Rathaus von Neswisch}

Der Ratsaal war der größte Raum des Rathauses und erfüllte eine Verwaltungsfunktion. Hier fanden die Sitzungen des Stadtrates sowie die wichtigsten Feiern der Stadt statt. Im Vorraum dieser Halle sind Kopien von Dokumenten über die Verleihung des Magdeburger Rechts und die Abschaffung des Rathauses nach der Eingliederung der Stadt Neswisch in das Russische Reich ausgestellt. Die Wände der Halle schmücken die Porträts von Menschen, die bedeutende Spuren nicht nur in der Geschichte und Kultur von Neswisch, sondern auch in der Geschichte des Großfürstentums Litauen und des Königreichs Polen (Rzeczpospolita) hinterlassen haben. Das sind die Porträts von Barbara Radziwill; Nikolai Radziwill, dem Schwarzen (pol. »Czarny«); Stephan Bathory; Nikolai Christophor Radziwill Sirotka; Giovanni Maria Bernardoni; Tomasz Makowski; Michail Kasimir Radziwill, 
der den Beinamen »Rybonka« trug; Ursula Franziska Radziwill; Karol Stanislaw Radziwill, genannt »Pane Kachanku« und Matthias Radziwill.

\section{Der Gerichtssaal im Rathaus von Neswisch}

Der Gerichtssaal im Rathaus von Neswisch befand sich links vom Ratsaal (Rada). Hier fanden die Gerichtsverhandlungen von zwei Kollegien des Magistrats statt. Das Bürgermeister- und Ratskollegium, an dessen Spitze ein Bürgermeister stand, verhandelte in Zivilsachen. Das Vogt- und Schöffenkollegium unter Leitung des Vogts beschäftigte sich mit Strafsachen sowie mit Sachen, bei denen eine der Parteien nicht unter einen Gerichtsstand der Stadt fiel. In der Ausstellung in diesem Gerichtssaal kann man die Kopien von Gerichtsprotokollen des Magistrats von Neswisch sehen, die einen Einblick in die Gerichtsordnung sowie in die Angelegenheiten, die in den Gerichtssitzungen im Rathaus von Neswisch verhandelt wurden, ermöglichen. Hier werden auch die Gegenstände gezeigt, die zur Bestrafung der Menschen dienten, die ein Verbrechen begangen hatten.

\section{Der Beratungsraum im Rathaus von Neswisch}

Der Beratungsraum befand sich immer neben dem Gerichtssaal. Hier traten der Vogt und die Schöffen zusammen, um sich vor dem Urteil zu beraten. Nach Meinung einiger Forscher war hier gleichzeitig das Stadtarchiv. Heute wird ein Teil der Aktenbücher des Magistrats in Neswisch (10 Einheiten für die Zeit von 1723-1795) im historischen Nationalarchiv Weißrusslands in Minsk aufbewahrt. Im Beratungsraum wurde ein Ausstellungskomplex gezeigt, der die Entwicklung von Gewerbe und Handel dokumentiert. Mit Hilfe einiger historischer Dokumente und Museumsstücke kann man sich einen Eindruck von Neswisch und von der Beschäftigung der Einwohner dieser Stadt im 17.-19. Jahrhundert verschaffen. Hier werden auch die Erzeugnisse von Handwerkern präsentiert, die bei archäologischen Ausgrabungen auf dem Stadtgebiet gefunden oder durch die Einwohner dem Museum übergeben wurden. Den zentralen Teil des Raums nimmt ein Stadtmodell ein, das die Stadt Neswisch am Ende des 18. Jahrhunderts bis zum Anfang des 19. Jahrhunderts zeigt.

\section{Die Schatzkammer im Rathaus von Neswisch}

Die Schatzkammer nahm in jedem Rathaus einen wichtigen Platz ein. In Neswisch war sie in den kleinen Räumen des Stadtmagistrats, hauptsächlich im Turm, untergebracht, und hieß »Skarbnitza«. Eine solche Unterbringung garantierte eine gewisse Sicherheit. Hier verwahrte man die Symbole der Stadtmacht und den Stadtschatz. In der Ausstellung im Rathaus von Neswisch ist dieser Raum im zweiten Stock des Turmes. Hier sind ein im Jahr 2002 im Stadtgebiet gefundener Münz-schatz, die Münzen- und Papiergeldsammlung sowie die mit der Finanztätigkeit des Magistrats von Neswisch verbundenen Urkunden ausgestellt. Darüber hinaus verwahrte man beim Schatz (»Skarbnitza«) im Rathaus teilweise, wenn möglich, den Güterbesitz armer Waisenkinder bis zu deren Volljährigkeit. Das Geld und die Wertsachen befanden sich in großen Truhen. 


\section{Das Arbeitszimmer des Vogts im Rathaus von Neswisch}

Der letzte Ausstellungsraum des Rathauses ist das Arbeitszimmer des Vogts, des Oberhaupts der Stadt. Der Vogt als Vertrauensperson eines Magnaten war dessen Vertreter und hatte bestimmte Rechte und Privilegien. Er war nicht nur der Vorsitzende des Magistrats, sondern stand auch an der Spitze des Gerichts. In Übereinstimmung mit dem Magdeburger Recht leistete der Vogt, wenn er sein Amt antrat, einen Eid, der es ihm zur Pflicht machte, zum Wohle der Stadt beizutragen. Der Vogt hatte das Recht, in allen Angelegenheiten der Stadt zu entscheiden. Wenn die Verwaltungsverordnungen und Entscheidungen des Vogts durch das gesamtstaatliche Gesetz und das Magdeburger Recht nicht gedeckt waren, war der Rat berechtigt, sie vor der Obergewalt anzufechten. Über die Verwaltungsarbeit in der Stadt Neswisch informieren im Arbeitszimmer des Vogts Abschriften von Urkunden, Photographien, Bücher und sonstige Gegenstände des 19. bis zum Anfang des 20. Jahrhunderts.

\section{Schlussfolgerung}

I. Wie ich in meinem Aufsatz ausgeführt habe, sind im Nationalarchiv in Minsk zur Rezeption des Sachsenspiegels und des Magdeburger Stadtrechts folgende Bestände vorhanden:

1) Die Bestände der städtischen Selbstverwaltungsorgane und Gerichtsbehörden (Magistrate, Stadt- und Burggerichte, Landgerichte usw.);

2) Familienbestände und Bestände zur persönlichen Abstammung;

3) Sammlungen (Urkundensammlungen sowie auch Sammlungen von Mikroverfilmungen von Urkunden).

II. Was die Nationalbibliothek in Minsk betrifft, wurden von mir, erstens, die Privilegien nach deutschem Recht; zweitens, die städtischen und gerichtlichen Statuten, Willküren, Plebisciten; drittens, die Gesetzbücher und Sammlungen des Magdeburger und Kulmer Rechts präsentiert.

III. Mein Bericht über den Besuch des Museums in Neswisch sollte Ihnen die physische Ausgestaltung des deutschen Rechts (des Sachsenspiegels und Magdeburger Stadtrechts) in baulicher Gestalt anschaulich machen.

Diese drei Beispiele dienen zur Verdeutlichung der Anwendung des deutschen Rechts in Weißrussland. 